\title{
De quoi le papier peint peut-il témoigner ?
}

Catalogue Roesz, exposition à la Galerie carrée, Paris, 2015 : L'origine à venir.

Jean-Louis Déotte

\section{OpenEdition}

Journals

Édition électronique

URL : http://journals.openedition.org/appareil/2267

DOI : 10.4000/appareil.2267

ISSN : 2101-0714

\section{Éditeur}

MSH Paris Nord

Référence électronique

Jean-Louis Déotte, « De quoi le papier peint peut-il témoigner? », Appareil [En ligne], Galerie, mis en ligne le 28 mai 2019, consulté le 30 juillet 2020. URL : http://journals.openedition.org/appareil/2267 . DOI : https://doi.org/10.4000/appareil.2267

Ce document a été généré automatiquement le 30 juillet 2020.

\section{(c) $)(1) \Theta$}

Appareil est mis à disposition selon les termes de la Licence Creative Commons Attribution - Pas d'Utilisation Commerciale - Pas de Modification 4.0 International. 


\section{De quoi le papier peint peut-il témoigner?}

Catalogue Roesz, exposition à la Galerie carrée, Paris, 2015 : L'origine à venir.

Jean-Louis Déotte

1 René Vinçon se demandait en 1999, dans Artifices d'exposition, pourquoi l'art contemporain ne pouvait être exposé que dans une boîte blanche. Sa réponse en désarçonna plus d'un : c'est le peintre Sironi qui en 1932, pour la première Exposition de la Révolution fasciste, fit l'éloge de « la pauvreté absolue et élémentaire ».

2 On oublie souvent que le fascisme italien entretint des rapports beaucoup plus complexes avec la modernité que le nazisme ou la contre-révolution stalinienne.

3 Que serait par exemple l'architecture déconstructiviste d'Eisenman sans la Maison du Fascisme édifiée par Terragni?

4 A contrario, d'excellents musées comme la National Gallery ont réussi à conserver un ahurissant papier peint vert pour présenter leurs chefs-d'œuvre.

5 Il en va de l'imposition symbolique du blanc comme de la critique de l'ornemental en architecture, à partir de Loos. Quand certains de nos meilleurs architectes tel que Nouvel doublent leurs édifices d'une paroi de verre détachée, comme pour la Fondation Cartier ou pour le Musée du quai Branly ${ }^{1}$, il faut bien reconnaître que certaines évidences, qui semblaient achever l'histoire de l'art, n'avaient qu'une valeur relative. Le mouvement moderne a réussi à nous faire croire que dans tous les domaines, de la peinture à l'architecture en passant par la musique (Adorno), l'histoire de l'art s'arrêtait avec lui parce qu'il en exhibait les principes (Greenberg). Or, comme toujours, il n'y a pas de fin de l'histoire.

6 Il ne s'agit pas d'ennoblir les préfixes «néo » ou "post » : le «façadisme » actuel ne consiste pas en une forme de réaction esthétique, mais en un approfondissement de l'essentiel.

7 Quand Germain Roesz double, redouble et amplifie le schéma répétitif et mécanique de papiers peints des années 1960-1970, où ce schéma est là tout en étant partiellement 
effacé, et en même temps surexploité dans son principe par un diagramme de cercles, de cernes carrés, etc., ce n'est pas par désir nostalgique, mais parce qu'il serait temps, après avoir relégué le « décoratif » en dehors du système des Beaux-Arts (comme c'est évident dans l'esthétique de Kant par exemple), de revenir sur une décision prise à la fin de l'art d'incarnation, en Occident médiéval. Quand peinture et sculpture ont cessé de répondre aux impératifs médiévaux théologico-politiques de la chrétienté, quand les Florentins ont accepté les leçons des Arabes, à savoir la rationalisation de la perception visuelle modelisée par l'usage de la camera obscura ${ }^{2}$ inventée par Al Hazen, en rupture avec une conception du milieu visuel diaphane héritée d'Aristote, la géométrisation du plan aurait pu s'imposer (pavage du plan) universellement, puisque la nouvelle peinture était en son fond, rationnelle.

8 Cette possibilité revient de temps à autre chez Roesz, en particulier quand, au cours de l'année 2003, après un séjour fructueux en Iran, il utilisa des nappes d'Ispahan comme support sur lequel des souvenirs de la peinture occidentale, du néolithique à la peinture «néo-géo » new yorkaise, étaient accueillis, en oubliant tous les différends cosmétiques.

Le différend cosmétique entre Islam et Occident, deux civilisations de la Parole révélée, s'oriente actuellement vers l'opposition entre deux théologies: celle de l'incarnation chrétienne, celle du « devenir livre » de l'Islam (« inlibration»). C'est se polariser sur l'iconophilie du christianisme et sur son refus dans l'Islam. Or Oleg Grabar ${ }^{3}$ relativise cette opposition, en notant, d'une part, que l'Islam chiite a préservé l'héritage persan de la miniature, d'autre part, en minimisant le refus islamique. Au moment où la question de l'image se serait posée pour l'Islam, Byzance était ravagée par les conflits entre iconoclastes et iconodules: il devenait prudent de ne pas s'engager sur un territoire si complexe et si mouvant. Marginalisant l'image, c'est-à-dire la fantasmagorie chrétienne, le choix se serait porté sur un art favorisant la contemplation du croyant par la géométrisation de l'espace interne des architectures et, d'une manière générale, des artefacts. Un rouleau encollé de 114 dessins d'architecture, conçu vers 1500 dans un milieu iranien, et conservé au musée de Topkapı à Istanbul, est de ce point de vue une mine :

Ces dessins d'architecture ne représentent pas des édifices mais des motifs pour l'ornement des murs et des modèles de voûtes pour la pratique architecturale. Ce répertoire de formes géométriques est universel, au point de faire abstraction des formes et de la dimension des constructions. Les architectes qui s'appuyaient sur ces modèles pouvaient concevoir et s'affranchir des solutions connues. La majorité des dessins est destinée à ce qu'on appelle des muquarnas.

Et Belting de conclure :

La Renaissance simule une image perçue (Sehbild) qui est liée au regard d'un individu et rapportée au lieu qu'il occupe. [...] C'est à travers la surface que l'on voit. La culture arabe, en revanche, considère la surface positivement. Elle est le lieu où une géométrie dotée d'une portée cosmique peut être représentée et calculée mathématiquement $t^{4}$.

10 Mais comme la notion de "surface » ou de medium est chez Belting floue ${ }^{5}$, il est amené à comparer des régimes cosmétiques qui dépendent de normes symboliques totalement étrangères. On ne peut pas simplement affirmer que les Arabes ont eu une conception positive de la surface et les Italiens de la Renaissance, «négative ». L'art musulman est suspendu à la révélation de la parole, l'art inventé à Florence à la projection et donc à la représentation de la nature. Ce qui suppose évidemment que la surface (du tableau, de la fresque ou du papier) ait été définie cosmétiquement (par Alberti) comme une 
surface projective. Ce sont donc des rapports à la loi, au symbolique, que tout oppose. À partir de cette décision inouïe, que personne en fait n'a prise, Dieu, la Madone, l'Enfant, les saints, etc. seront mis au carreau comme tout le reste. C'est ce qu'on appelle, d'une manière erronée, « humanisme », comme si le monde de la représentation était arrimé à l'homme, alors que c'est le contraire. L'homme de l'Humanisme n'est qu'une représentation, subissant une loi identique : celle de la rationalisation de l'espace.

11 Si Belting a raison d'insister sur l'apport géométrique des Arabes (le zéro est une sorte de préfiguration du « point de fuite » perspectif), il s'interdit de penser l'extraordinaire productivité de l'appareil perspectif qui vient appareiller la norme projective, et ce jusqu'au tournant du $\mathrm{xIX}^{\mathrm{e}}$ avec les Impressionnistes, Monet, Cézanne, Manet, etc. Au contraire, une fois atteintes, les relations géométriques des muquarnas immobilisèrent les formes de l'art musulman. Mais elles perdurèrent en sous main dans l'art « décoratif » occidental.

Dès lors, l'assimilation puis la persistance du « décoratif » dans l'art occidental doivent être pensées comme la trace sous-jacente du rapport à la révélation dans son acception musulmane, et non comme le simple rejet social d'un art "mécanique », "répétitif ", témoignant d'une simple production artisanale.

Il est évident que la manière esthétique de laisser revenir le " décoratif », chez Matisse par exemple ou chez les Viennois, a été rendue possible à l'époque du musée, lequel comme appareil, suspend toutes les destinations cosmétiques, qui deviennent dès lors comparables.

14 Finalement, d'une manière discrètement engagée, Roesz intervient dans le débat actuel, terriblement instrumentalisé sur le plan politique ${ }^{6}$. Entre ceux qui utilisent la liberté d'expression pour que l'adversaire islamiste se dévoile dans sa barbarie et ceux qui pratiquent une sorte d'archaïsme indissociable de la mondialisation où la foi et les médias s'interpénètrent, Roesz et sa nouvelle série picturale rappellent qu'il n'y aurait pas eu de peinture occidentale sans la géométrisation musulmane de la vision, et que cette dernière n'a pas cessé de l'habiter, mais marginalisée, ou mieux comme fairevaloir insignifiant : le « décoratif ».

\section{BIBLIOGRAPHIE}

Sandrine Amy : «Mur rideau ; la façade en jeu », in Appareil, $\mathrm{n}^{\circ}$ 11, 2013, https://

appareil.revues.org/1761

Hans Belting : Florence et Bagdad. Une histoire du regard entre Orient et Occident, Paris, 2012, trad. fr. A. Rieber et N. Ghermani.

Hans Belting, Pour une anthropologie des images, tard. fr. Jean Torrent, Paris, Gallimard, coll. « Le Temps des images », 2004

Oleg Grabar, Penser l'art islamique. Une esthétique de l'ornement, Paris, Albin Michel, coll. « La Chaire de l'IMA », 1996. 


\section{NOTES}

1. Voir l'étude de Sandrine Amy: "Mur rideau; la façade en jeu », in Appareil, $\mathrm{n}^{\circ} 11,2013$, https://appareil.revues.org/1761

2. Hans Belting: Florence et Bagdad. Une histoire du regard entre Orient et Occident, Paris, 2012, trad. fr. A. Rieber et N. Ghermani.

3. Oleg Grabar, Penser l'art islamique. Une esthétique de l'ornement, Paris, Albin Michel, coll. « La Chaire de l'IMA », 1996.

4. Hans Belting, Ibid., p. 158-160.

5. Hans Belting, Pour une anthropologie des images, tard. fr. Jean Torrent, Paris, Gallimard, coll. « Le Temps des images ", 2004.

6. Carsten Juhl est professeur d'esthétique à l'Académie royale des Beaux-arts de Copenhague : < $>$. 